\title{
Distributed Fair Transmit Power Adjustment for Vehicular Ad Hoc Networks
}

\author{
Marc Torrent-Moreno \\ Institute of Telematics \\ University of Karlsruhe \\ Germany \\ torrent@tm.uni-karlsruhe.de
}

\author{
Paolo Santi \\ Istituto di Informatica \\ e Telematica del CNR \\ Pisa, Italy \\ paolo.santi@iit.cnr.it
}

\author{
Hannes Hartenstein \\ Institute of Telematics \\ University of Karlsruhe \\ Germany \\ hartenstein@rz.uni-karlsruhe.de
}

\begin{abstract}
Improving the safety of drivers and passengers by wirelessly exchanging information between vehicles represents a major driving force for the design of vehicular ad hoc networks. In a heavy loaded 802.11-based network, however, safety-related packets might collide frequently and cannot be decoded by a receiver, thus they might not be effective in increasing the safety level on the roads. In this paper, we propose to use transmit power control in order to reduce packet collisions, while taking into account the major design goal of vehicular ad hoc networks, i.e. increasing safety. While previous work has addressed the issue of power control primarily for optimizing network capacity and/or connectivity, the optimization criterion for improving safety has to be built upon the concept of fairness: a higher transmit power of a sender should not be selected at the expense of preventing other vehicles to send/receive their required amount of safety information. In this paper, we propose a fully distributed and localized algorithm called D-FPAV (Distributed Fair Power Adjustment for Vehicular networks) for adaptive transmit power adjustment which is formally proven to achieve max-min fairness. Furthermore, we investigate the effectiveness and robustness of D-FPAV through extensive simulations based on a realistic highway scenario and different radio propagation models.
\end{abstract}

\section{INTRODUCTION}

The number of fatalities on public roads is a main concern for both public opinion and country's governments. Several initiatives [1] have been started with the objective of significantly decreasing both the number of accidents and their resulting damage. These efforts do not only consider a better consciousness of drivers and an adequate road system, but also the use of new technologies capable of assisting drivers in order to improve safety conditions.

Among the new technologies considered, vehicular ad hoc networks (VANETs) play an important role, since the use of wireless communications offer the beneficial capability of directly exchanging safety-related information between vehicles. Various efforts (projects such as VII [2], C2CCC [3], InternetITS [4], etc., and standard bodies such as IEEE [5]) are currently developing a technology that combines 802.11based wireless communications with on-board sensors (e.g., GPS, speedometers) in order to improve the driver's awareness of the surrounding environment, making available information which he/she or other on-board sensors (e.g., radar) might not be able to 'see'.

The exchange of safety-related information comes into two flavors: $i$ ) by detecting potentially dangerous situations through the periodic exchange of status information (i.e., broadcast beacons containing position, speed and so on) between all vehicles in the surrounding, and ii) by rapidly disseminating hazard warnings in case of an emergency (event-driven messages).

In this context, a major challenge is related to resource allocation among the network participants: when VANETs will be fully deployed, medium to high traffic densities will result in heavy-load channel conditions. In such situations (that are critical from the safety point of view), given the broadcast nature of the exchanged information, a high number of packet collisions is expected. Thus, the minimum amount of required data needed to provide adequate safety-level from an information point of view might not be reached.

A possible way of mitigating this problem is to introduce strategies to control the channel load that explicitly take into account the major goal of VANETs, i.e. increasing the safety conditions on the road.

In this paper, we propose a fully distributed strategy to control the channel load based on adjusting the nodes' transmit power in order to prevent packet collisions that could excessively degrade the safety-related information exchange. Our technique is built upon the concept of fairness, which we believe is fundamental in order to achieve VANETs' final goal of increasing safety. As we thoroughly discuss in the next section, previously proposed power control approaches aimed at optimizing network capacity and/or connectivity do not suit VANETs' characteristics. The fundamental observation is that, to make safety applications capable of detecting an unsafe situation and taking the right decisions to avoid a danger in case of emergency, it is very important that every vehicle has a good estimation of the state of all vehicles (with no exception) in its closer surrounding. In other words, if a vehicle is not assigned a fair portion of the resources, it can not announce itself to its closer neighbors, and becomes a danger itself. Thus, the available channel capacity must be shared among nodes in a fair way.

The fully distributed strategy introduced in this paper, called D-FPAV (Distributed Fair Power Adjustment for Vehicular Networks), is explicitly designed taking VANETs characteristics into account, and it is formally proven to achieve fairness, and to balance the relevance between beacon and 
event-driven messages. The effectiveness of D-FPAV has been verified through ns-2-based simulations with realistic highway traffic patterns and various radio propagation models. The results show that the use of D-FPAV significantly increases the probability of receiving safety messages in case of emergency compared to the case of no power control.

The remainder of this paper is organized as follows. In Sec. II, we discuss the motivations to study the problem at hand. Sec. III presents the most relevant work related to the discussed issue. In Sec. IV, we introduce the D-FPAV distributed algorithm for computing a provably fair power assignment. Sec. V presents the results of the simulation study performed to assess the effectiveness of our proposed power control strategy. Finally, Sec. VI concludes the paper.

\section{RATIONALE}

\section{A. Assumptions and Observations}

In accordance with the FCC ruling report [6] we assume that the lower layer technology used in VANETs will be a variant of IEEE 802.11a, now IEEE 802.11p [7], and there will be one $10 \mathrm{MHz}$ control channel primarily for the exchange of safety-related messages. The Distributed Coordination Function (DCF), i.e., the IEEE 802.11 basic access mechanism, is a totally asynchronous approach. DCF is known for its inability to efficiently manage the medium resources, especially in case of broadcast messages. A $10 \mathrm{MHz}$ channel only offers half the data rates of 802.11a, and lower rates are preferred because of their robustness to noise and interference [8]. Finally, we can expect safety-related messages to be relatively large due to the security overhead: recent studies [9] report numbers between 250 and 800 Bytes for the message size.

With a configuration as described above, [10] presents a simulation work showing the severe impact of the well-known hidden terminal problem due to the resulting packet collisions in broadcast scenarios.

In such challenging conditions communication protocols should aim to satisfy safety applications requirements or, in communication terms, keep packet collisions in the vehicle surroundings low. As outlined in the previous section, safety applications will not only transmit periodic messages in the control channel, but also event-driven ones. Therefore, a key aspect for the design of appropriate communication protocols is to clarify what type of messages is more important depending on the distance to the sender. The purpose and relevance of both types of messages are described in the following.

Periodic messages can be seen as preventive messages in terms of safety. They convey information about the state of the sending vehicle, i.e., position, direction, speed, etc., and possibly also aggregated data regarding the state of its neighbors. It is reasonable to assume that these periodic messages (also called beacons in the following) will be sent in a broadcast fashion since the messages' content can be beneficial for all vehicles around. Note that the existence of a beaconing mechanism can be fundamental also to implement non-safety applications (e.g., traffic monitoring), or protocols (e.g., geocasting). The main goal of beaconing is to improve driver awareness of the surrounding environment. We remark that even though the reception of a beacon is somehow 'expected', the information contained can be of critical importance in close distances to the sender since it can make possible to detect an unsafe road situation (i.e., proximity of crossing vehicles at an intersection). This argumentation line is followed in other studies, e.g. [11], to suggest that the beaconing message generation rate should be in the order of several packets per second to provide the safety system with accurate enough information from the close surrounding.

Event-driven messages, instead, are the result of the detection of a hazard, e.g., hard braking from a car, an emergency vehicle driving at high speed, etc. Hence, the information they convey is of primary importance also for relatively farther nodes, and they should be propagated as quickly as possible along the road. The purpose of event-driven messages is to enable drivers to undertake adequate countermeasures in case of an emergency: several accidents could be avoided if drivers would be made aware of the peril just few seconds (or even a fraction of a second) before they can actually see it.

\section{B. What is needed?}

Fully deployed VANETs will encounter situations where the technology limitations described above become a challenge. Unfortunately, these situations are often critical in terms of safety. For instance, consider scenarios with medium to high vehicle densities and relatively high speeds, such as highways near the entrance of big cities or a temporary working area. Due to a large number of vehicles sharing the medium, it is not clear whether the channel capacity is sufficient to support the data load generated by beaconing while at the same time leaving enough available bandwidth for event-driven safety messages.

In the following we outline the rationale behind our strategy in linking safety and fairness as a sequence of reasonings that eventually define the requirements for fair power adjustment for vehicular networks.

1. Relevance of safety messages. Event-driven messages should be able to access the control channel with short delay, and they should have low probability of collision even when targeting large areas, i.e., when being transmitted with high power. Beacons, on the other hand, show a high relevance in the close neighborhood of the sender, but they are less relevant at higher distances (in analogy to the standard 'safety distance' of vehicles). Thus, a resource allocation strategy is needed that achieves a clear prioritization, or balance, among the messages according to their relevance for safety.

2. Balancing event-driven messages and beacons. Taking into account the relevance for safety, it is our belief that the amount of load resulting from beaconing should be limited: it is desirable to $i$ ) avoid a high number of beacon's collisions, and ii) leave some available bandwidth to handle unexpected emergency situations with the necessary reliability. Thus, we need to design a 'congestion control mechanism' which is able to keep the periodic messages' load under a specific maximum value at all the nodes of the network. This threshold, called 
MaxBeaconingLoad (MBL) in the following, represents a limit where safety protocols can achieve a reasonable performance at a specified data rate (or modulation).

3. Keeping the beaconing load below MBL. We propose to adjust the transmission power used for beaconing messages in order to keep the load in the medium below MBL. This way, by using the design proposed in this paper, the MBL threshold can be seen as a handle to fine-tune the level of prioritization between beacon and event-driven messages: by increasing the MBL, the beaconing activity is assigned a larger portion of the available bandwidth, and a relatively "lower" priority (although still higher than that of beacons) is implicitly assigned to event-driven messages. In this paper, we assume that the MBL threshold is assigned a fixed level (set to half of the available bandwidth in Section V), and we leave the design of a strategy for dynamically setting the MBL value, e.g., depending on traffic conditions, as future work.

We are aware that before decreasing the transmission power of beacons other strategies should be implemented, such as realizing an admission control mechanism to drop all nonsafety related packets before being sent to the control channel, or dynamically adjusting the packet generation rate. Nevertheless, there will be many situations where decreasing the transmission range of certain nodes on top of that is necessary (e.g., fast moving medium density traffic conditions). Although transmit power control has been a deeply studied subject in the mobile networks field already (see related work in Section III), vehicular environments present new challenges. Most of previous studies addressing power control try to ensure connectivity and/or optimize capacity of ad hoc networks. However, the primary goal of transmit power control when applied to VANETs is not to optimize data transport capacity for several ongoing point-to-point communications, or to build a connected network topology (see the toy examples depicted in Fig. 1), but instead to improve as much as possible the driver's awareness of a vehicle's surroundings.

4. The elements of fairness. To limit the beaconing load offered to the medium at all points in the network below the specific common MBL, vehicles should restrict their beacons' transmission power. From an individual safety point of view (e.g., looking at the kinetic energy of vehicles) a higher range of awareness is preferable. From a system perspective the vehicle with the minimum transmission power can be considered a safety hazard for the other cars (or vice versa). As already observed in the introduction, it is very important that every node (vehicle) has a good estimation of the state of all vehicles (with no exception) in its closer surrounding. Thus, essentially, a max-min fairness concept is required. One might argue that due to different velocities of vehicles - or general due to different 'states' of the vehicles - the vehicles should send beacons with transmission power related to their velocity or status. In this case the transmission power should be restricted by the same ratio to satisfy MBL.

5. Fairness with low complexity. Additionally, vehicular networks are composed of highly mobile nodes. Therefore, the power adjustment mechanism cannot be based on a strategy

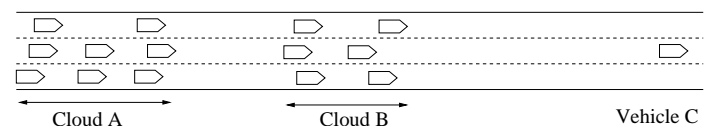

(a) If nodes from Cloud B would try to be connected with far away Vehicle $\mathrm{C}$, they can create high interferences disturbing information exchange among vehicles inside Cloud A.

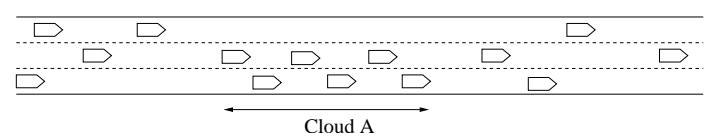

(b) If nodes forming Cloud A would try to optimize capacity they would adjust their transmit power to reach only the closest car. In this case, they would not have direct awareness of the next vehicle in the same lane even though it is very close.

Fig. 1. Road situations showing drawbacks of unfair power control in VANETs.

which converges to stable power settings over a relatively long period of time; instead, it must be able to quickly react to changes of nodes' requirements and locations.

\section{RELATED WORK}

This paper is the continuation of our work [12], where we introduced the concept of fairness as a key factor for vehicular safety communications. The paper [12] also presented a strategy to achieve a max-min fairness power assignment (inspired by Water Filling as presented in [13]) assuming global knowledge, i.e., using a centralized algorithm, which is clearly impractical in a vehicular environment. The contributions of the present paper with respect to [12] are: $i$ ) a fully distributed scheme to achieve the fair power assignment, which is formally proven to achieve fairness, ii) a strategy to estimate the state of a vehicle's surrounding, needed for the proper functionality of the power assignment technique, and iii) extensive simulations, with three different radio propagation models, that show how the proposed strategies accomplish the goals presented in the previous section in real highway scenarios.

Apart from [12], the previous work related to this paper can be classified in two main categories. The first one is topology control in ad hoc networks. Although it has been an intensively studied field for many years, VANETs' specific paradigms make all these analyses or proposed algorithms not valid to satisfy their requirements. Most of these studies deal with point-to-point communications and try to find a path to destination with a local or distributed approach while maximizing the system overall capacity and/or energy consumption ([14] and [15]). As outlined in Sec. II, neither overall capacity nor energy efficiency are the main driving factors in VANETs. Maybe, the study in this area which is most related to our work is [16], which proposes an adaptive algorithm to maximize 1-hop broadcast coverage. However, this study addresses static networks and does not consider different requirements with respect to communication ranges, which makes their approach not valid for VANETs. 
The second area of research related to our work is the one addressing fairness to share the wireless medium. In this category we can find strategies that consider only unicast communications and $i$ ) assign a portion of the estimated bandwidth to each flow, such as [17], or ii) provide a scheduling mechanism to achieve its fairness criteria, e.g., [18].

Recently, due to the increasing attention of researchers on VANETs, some studies have tried to apply these methodologies to vehicular environments. For example, [19] addresses power control in VANETs with the goal of producing a connected network topology; or [20], which describes a scheme based on a utility fair function to share the broadcast medium. In the latter, a scheduling approach is proposed that can be perfectly valid for non-safety VANETs' applications, however, it does not satisfy all the safety constraints presented in Sec. II.

\section{The Distributed FPAV ALgorithm}

Based on the rationale outlined in Section II and on the BMMTxP (Beaconing Max-Min Tx Power) problem as given in [12] we define the D-FPAV algorithm and formally prove that it achieves fairness.

\section{A. The BMMTxP problem and D-FPAV}

Here, a short version of the problem statement and definitions presented in [12] are reproduced, which are required to prove Theorem 1. We refer the reader to Section 3.2 of [12] for a complete explanation with the correspondent justifications.

Assume a set of nodes $N=\left\{u_{1}, \ldots, u_{n}\right\}$ is moving along a road modeled as a line ${ }^{1}$ of unit length, i.e. $R=[0,1]$, and that nodes can be modeled as points $x(i) \in[0,1]$.

Each of the network nodes sends a beacon with a predefined beaconing frequency $F$, using a certain transmit power $p \in\left[0, P_{\max }\right]$.

Definition 1 (Power assignment): Given a set of nodes $N=\left\{u_{1}, \ldots, u_{n}\right\}$, a power assignment $P A$ is a function that assigns to every network node $u_{i}$, with $i=1, \ldots, n$, a ratio $P A(i) \in[0,1]$. The power used by node $u_{i}$ to send the beacon is $P A(i) \cdot P_{\max }$.

Definition 2 (Carrier Sensing Range): Given a power assignment $P A$ and any node $u_{i} \in N$, the carrier sensing range of $u_{i}$ under $P A$, denoted $C S(P A, i)$, is defined as intersection between the commonly known CS range ${ }^{2}$ of node $u_{i}$ at power $P A(i) \cdot P_{\max }$ and the deployment region $R$. The CS range of node $u_{i}$ at maximum power is denoted $C S_{M A X}(i)$.

Given a power assignment $P A$, the network load generated by the beaconing activity under $P A$ is defined as follows.

Definition 3 (Beaconing load under PA): Given a set of nodes $N$ and a power assignment $P A$ for the nodes in $N$,

\footnotetext{
${ }^{1}$ Modeling the road as a line is a reasonable simplification in our case since we assume the communication ranges of the nodes to be much larger than the width of the road.

${ }^{2}$ The CS (Carrier Sense) range, in ideal conditions, is the distance to which a node's transmissions can be sensed, or also considered as the distance to which a node can interfere with other transmissions.
}

\begin{tabular}{|c|c|c|c|}
\hline$\stackrel{\bullet}{u_{1}} \quad u$ & 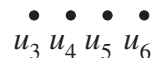 & $\ddot{u_{7}}$ & $\dot{u}_{8}^{\bullet}$ \\
\hline
\end{tabular}

Fig. 2. Node deployment used in the example of D-FPAV execution.

the beaconing network load at node $u_{i}$ under $P A$ is defined as:

$$
B L(P A, i)=\left|\left\{u_{j} \in N, j \neq i: u_{i} \in C S(P A, j)\right\}\right|,
$$

where $C S(P A, j)$ is the carrier sensing range of node $u_{j}$ under power assignment $P A$.

We remark that the above definition of beaconing load can be easily extended to account for beacon messages of different size, and for different beaconing frequencies in the network. The framework for distributed power control discussed below can be applied also with a more general definition of beaconing load.

Definition 4: (Beaconing Max-Min Tx power Problem $(B M M T x P)):$ Given a set of nodes $N=\left\{u_{1}, \ldots, u_{n}\right\}$ in $R=[0,1]$, determine a power assignment $\overline{P A}$ such that the minimum of the transmit powers used by nodes for beaconing is maximized, and the network load experienced at the nodes remains below the beaconing threshold $M B L$. Formally,

$$
\left\{\begin{array}{l}
\max _{P A \in \mathbf{P A}}\left(\min _{u_{i} \in N} P A(i)\right) \\
\text { subject to } \\
B L(P A, i) \leq M B L \forall i \in\{1, \ldots, n\}
\end{array},\right.
$$

where PA is the set of all possible power assignments.

In [12], we presented $F P A V$, an algorithm based on 'water filling' able to compute $P A$ when global knowledge was assumed. In order to facilitate reading and due to completeness we describe the essentials of the FPAV algorithm. Fig. 3 presents FPAV which works as follows: every node starts with the minimum transmit power assigned; then, all the nodes increase their transmit power simultaneously of the same amount $\epsilon \cdot P_{\max }$ as long as the condition on the beaconing network load (MBL) is satisfied. At the end of FPAV all nodes have increased their power the same number of steps $\mathrm{k}$ and end up with a power of $p=(k \epsilon) \cdot P_{\max }$. Note that in [12] we also proposed a 'second stage' of the FPAV algorithm in order to achieve per-node maximality. However, it is not included in the present study because further simulations showed a marginal gain in many cases due to the high network dynamics. In the following we present a fully distributed, asynchronous, and localized algorithm called D-FPAV for solving BMMTxP.

D-FPAV is summarized in Figure 4. A node $u_{i}$ is continuously collecting the information about the status (current position, velocity, direction, and so on) of all the nodes within its carrier sensing range at maximum power $C S_{M A X}(i)$. These are the only nodes that node $u_{i}$ can affect when sending its beacon. The actual mechanism to acquire the required state information is discussed in the following subsection. Based on this information, node $u_{i}$ makes use of FPAV to compute the maximum common value $P_{i}$ of the transmit power for all 


\section{Algorithm FPAV:}

INPUT: $\quad$ a set of nodes $N=\left\{u_{1}, \ldots, u_{n}\right\}$ in $[0,1]$

OUTPUT: a power assignment $P A$ which is an

$\left(\epsilon \cdot P_{\max }\right.$-approximation of an) optimal solution to BMMTxP

$$
\begin{aligned}
& \forall u_{i} \in N \text {, set } P A(i)=0 \\
& \text { while }(B L(P A) \leq M B L) \text { do } \\
& \quad \forall u_{i} \in N, P A(i)=P A(i)+\epsilon \\
& \text { end while } \\
& \forall u_{i} \in N, P A(i)=P A(i)-\epsilon
\end{aligned}
$$

Fig. 3. The FPAV algorithm.

\section{Algorithm D-FPAV: (algorithm for node $u_{i}$ )}

INPUT: $\quad$ status of all the nodes in $C S_{M A X}(i)$

OUTPUT: a power setting $P A(i)$ for node $u_{i}$, such that the resulting power assignment is an optimal solution to BMMTxP

1. Based on the status of the nodes in $C S_{M A X}(i)$, compute the maximum common tx power level $P_{i}$ s.t. the MBL threshold is not violated at any node in $C S_{M A X}(i)$

$2 a$. Broadcast $P_{i}$ to all nodes in $C S_{M A X}(i)$

$2 b$. Receive the messages with the power level from nodes $u_{j}$ such that $u_{i} \in C S_{M A X}(j)$; store the received values in $P_{j}$

3. Compute the final power level: $P A(i)=\min \left\{P_{i}, \min _{j: u_{i} \in C S_{M A X}(j)}\left\{P_{j}\right\}\right\}$

Fig. 4. The D-FPAV algorithm.

nodes in $C S_{M A X}(i)$ such that the condition on the MBL is not violated (step 1). Note that this computation is based on local information only (the status of all the nodes in $C S_{M A X}(i)$ ), and it might be infeasible (i.e., it might violate the condition on MBL at some node) globally. To account for this, node $u_{i}$ broadcasts the computed common power level $P_{i}$ to all nodes in $C S_{M A X}(i)$ (step 2a). In the meanwhile, node $u_{i}$ receives the same information from the nodes $u_{j}$ such that $u_{i} \in C S_{M A X}(j)$ (step 2b). After having received the power levels computed by the nodes in its vicinity, node $u_{i}$ can compute the final transmit power level, which is set to the minimum among the value $P_{i}$ computed by the node itself and the values received from nodes in the vicinity (step 4). Setting the final power level to the minimum possible level is necessary in order to guarantee the feasibility of the computed power assignment (see theorem below).

Theorem 1: Assume the CS ranges of the nodes are symmetric, i.e. $u_{i} \in C S_{M A X}(j) \Leftrightarrow u_{j} \in C S_{M A X}(i)$. Then, algorithm D-FPAV computes an optimal solution to BMMTxP.
Proof: First, we have to show that the power assignment computed by D-FPAV is a feasible solution to BMMTxP. Assume the contrary, i.e. assume there exists node $u_{i}$ such that $B L(P A, i)>M B L$, where $P A$ is the power assignment computed by D-FPAV. This means that node $u_{i}$ has too many interferers, all of which are located in $C S_{M A X}(i)$ (assuming symmetric CS ranges). Let $u_{j}, \ldots, u_{j+h}$, for some $h>0$, be these interferers, and let $P A_{i}$ be the power assignment computed by node $u_{i}$ for all the nodes in $C S_{M A X}(i)$. In step 1 of D-FPAV, $u_{i}$ computes an optimal solution $P A_{i}$ to BMMTxP restricted to $C S_{M A X}(i)$. Assuming symmetric CS ranges, this solution includes a power setting for the interferers $u_{j}, \ldots, u_{j+h}$, and this power setting is such that $B L\left(P A_{i}, i\right) \leq M B L$. At step 2 of D-FPAV, the power setting $P A_{i}$ is broadcasted to all the nodes in $C S_{M A X}(i)$, which includes all the interferers $u_{j}, \ldots, u_{j+h}$. Hence, each of the interferers receives from node $u_{i}$ a power setting $P A_{i}$ such that the condition on the beaconing load is not violated at node $u_{i}$. Since the final power setting of the interferers is at most $P A_{i}$ (this follows from the minimum operation executed at step 3 of D-FPAV), and assuming a monotonic CS range, we have that the beaconing load at node $u_{i}$ cannot exceed the MBL threshold - contradiction. This proves that the power assignment computed by D-FPAV is a feasible solution to BMMTxP.

Let us now prove that the computed power assignment is optimal. Let $P A$ be the power assignment computed by D-FPAV, and let $p_{\min }$ be the minimum of the node power levels in $P A$. Assume $P A$ is not optimal, i.e. that there exists another feasible solution $P A^{\prime}$ to BMMTxP such that the minimum of the node power levels in $P A^{\prime}$ is $p^{\prime}>p_{\text {min }}$. Without loss of generality, assume that $P A^{\prime}$ sets the power level of all the nodes to $p^{\prime}$. Since $P A^{\prime}$ is feasible, we have that $B L\left(P A^{\prime}, i\right) \leq M B L \forall i \in 1, \ldots, n$. Hence, every node $u_{i}$ in the network computes a power setting $P_{i} \geq p^{\prime}$ at step 1 of D-FPAV. Consequently, the final power setting of every node in the network as computed by D-FPAV is at least $p^{\prime}>p_{\min }$, which contradicts our initial assumption. It follows that the solution computed by D-FPAV is optimal.

Theorem 2: Algorithm D-FPAV has $O(n)$ message complexity.

The straightforward proof of the theorem is omitted.

Let us use the scenario of Figure 2 to present D-FPAV execution with a toy example. We have eight cars, denoted $u_{1}, \ldots, u_{8}$, which are placed on a $1 \mathrm{~km}$ long road, with relative distance varying from $50 \mathrm{~m}$ to $200 \mathrm{~m}$. For the sake of clarity, we assume that the carrier sensing range can be represented as a segment centered at the transmitting node, and that the maximum CS range is initially $400 \mathrm{~m}$. We also assume that all nodes send beacons of the same size with equal frequency, being the maximum beaconing load MBL such that any node can be in the CS range of at most two other nodes.

We summarize D-FPAV execution with the matrix reported in Table I, where the row corresponding to node $u_{i}$ reports the values of the transmit power (actually, what is reported in 


\begin{tabular}{c|cc} 
& $u_{1}$ & \\
\hline$u_{1}$ & 150 & 150 \\
$u_{2}$ & 50 & \\
$u_{3}$ & 50 & \\
$u_{4}$ & 50 & \\
$u_{5}$ & 50 & \\
$u_{6}$ & \\
$u_{7}$ & \\
$u_{8}$ &
\end{tabular}

$u_{2}$
150
50
50
50
50
50

$\begin{array}{cc}u_{3} & u_{4} \\ 150 & 150 \\ 50 & 50 \\ 50 & 50 \\ 50 & 50 \\ 50 & 50 \\ 50 & 50 \\ 50 & 50\end{array}$

$u_{5}$
150
50
50
50
50
50
50
150

TABLE I

SuMMARIZATION OF D-FPAV EXECUTION. ENTRIES REPRESENT IN METERS THE MAXIMUM ALLOWED VALUE OF THE CS RANGE PER NODE.

the entries is the value of the CS range, which is defined by a correspondent transmit power level) as computed by node $u_{i}$ for all the nodes within its maximum CS range. For instance, the first row of the matrix represents the fact that node $u_{1}$ computes maximum allowed CS range equal to $150 \mathrm{~m}$ for all the nodes within its maximum CS range, i.e. nodes $u_{1}, \ldots, u_{5}$. On the other hand, columns represent all the values of the allowed CS range that a node receives from the nodes within its CS range. For instance, the first column represents the fact that node $u_{1}$ receives a power setting for itself by nodes $u_{2}, \ldots, u_{5}$. Then, the final power setting for every node in the network as computed by D-FPAV corresponds to taking the minimum over the values in its column. In our example, every node will end up D-FPAV execution setting the transmit power to a value such that the corresponding CS range is $50 \mathrm{~m}$.

\section{B. Estimation of status information}

Let us now discuss how node $u_{i}$ can collect the status information of all its surrounding nodes in order to compute the appropriate power level.

The only option to acquire status information from all nodes in $C S_{M A X}(i)$, which includes nodes located outside of the transmission range, is making use of a strategy where intermediate nodes re-transmit the status of their neighbors. Clearly, in a dynamic scenario only an estimation of the actual status information will be available at the time D-FPAV has to adjust the transmission power of a beacon, i.e., the last received information from neighboring nodes. Therefore, the accuracy of D-FPAV will depend on the frequency that each node transmits both its own and its neighbors status information. Furthermore, our algorithm requires the $P_{i}$ values from all nodes in $C S_{M A X}(i)$.

We propose that each node aggregates the $P_{i}$ values with the status information of the corresponding nodes inside $C S_{M A X}(i)$ and then, to improve efficiency, piggyback this aggregated information in beacon messages.

Now, decisions must be done with respect to what fraction of beacons should be piggybacked, and which transmit power should be used to send beacons containing this additional information. In making these choices there is a trade off between amount of load offered to the channel and accuracy of the neighbors status information available at the nodes. Utilizing a higher transmission power and piggybacking a higher number of beacons could provide a higher accuracy of the required information and, therefore, of D-FPAV performance. However, this additional amount of load have to be, in turn, controlled due to D-FPAV's purpose of limiting the load on the channel. In order to select the better option, we have performed a preliminary set of ns-2 based simulations, using five different configurations:

1) piggyback the aggregated status information to each beacon and transmit it with power $P A(i)$ (the transmit power value as computed by D-FPAV).

2) piggyback the information every 5 beacons and use power $P_{\max }$ for sending the augmented beacon.

3) piggyback the information every 5 beacons and use power $P A(i)$ for sending the augmented beacon.

4) piggyback the information every 10 beacons and use power $P_{\max }$ for sending the augmented beacon.

5) piggyback the information every 10 beacons and use power $P A(i)$ for sending the augmented beacon.

We considered that sending piggybacked beacons with a lower frequency than one every 10 beacons would cause D-FPAV to deal with too outdated information.

Simulation results, which are not shown due to lack of space, showed that option 5) (send the status every 10 beacons with power $P A(i)$ ) offers the best tradeoff between updated status information and additional overhead on the medium. For this reason, in the following we assume that neighbor status information is updated according to strategy 5) above.

\section{Simulation Study}

\section{A. Simulation Setup}

The version of the network simulator utilized in our experiments is ns-2.28 [21], although several improvements have been included to the downloadable version. First, several bugs have been fixed in the MAC layer [22]. Second, the physical layer has been adjusted to the IEEE 802.11p technology [7] with the values provided in [10]. Third, the Nakagami radio propagation model, whose parameters were adjusted to match actual measurements reported in [23], has been used.

In order to have a realistic scenario with a dynamic network topology, we employed the publicly available German highway patterns described in [24]. The chosen set for our evaluation is the most critical for safety: fast moving, 'heavy' traffic. In particular, we considered a $12 \mathrm{~km}$ long road with high traffic density, where vehicles travel at an average speed of $121.86 \mathrm{~km} / \mathrm{h}$. This straight portion of highway is composed of 6 lanes, 3 in each direction, with an average vehicle density of 11 vehicles per kilometer and lane (see a screenshot of the visualization tool HWGui [24] in Fig. 5).

There are several other parameters that have to be configured to run reasonable simulation scenarios. We selected a packet generation rate for beacons of 10packets/s, which is considered an acceptable value to provide accurate enough information to the safety system [11]. The packet size of all beacons have been fixed to 500Bytes, which is approximately in the middle of the interval of reasonable packet sizes in 


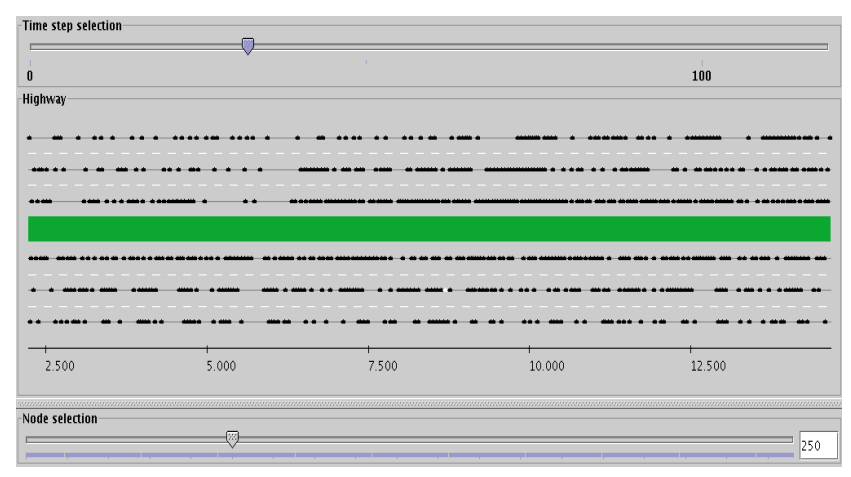

Fig. 5. Screenshot of the utilized scenario, which consists in a $12 \mathrm{~km}$ long bi-directional highway with 3 lanes per direction.

VANETs as reported in the security study [9]. In [8], it is shown that BPSK modulation schemes of OFDM-based wireless LAN technologies, such as $802.11 \mathrm{p}$, are advisable due to their robustness. We have chosen a $3 \mathrm{Mbps}$ data rate due to its lowest SNR requirement, namely $4 \mathrm{~dB}$. Concerning D-FPAV implementation, we have fixed the $M B L$ to $1.5 \mathrm{Mbps}$ (at most half of the channel capacity can be used for beaconing) and each neighbor entry to 15Bytes (corresponding to VehicleID and position). Finally, in order to see the effect of D-FPAV on different safety requirements, we have considered two intended communication ranges at maximum power, namely $250 \mathrm{~m}$ and $500 \mathrm{~m}$. With these configuration parameters, the maximum CS range is $397 \mathrm{~m}$ and $664 \mathrm{~m}$, respectively.

In order to see the effect that different radio propagation models have on D-FPAV performance, we have considered three well-known models as a representative set: the deterministic Two-Ray Ground (TRG) model, and the probabilistic Log-Normal Shadowing (LNS) and Nakagami (Nak) models. The TRG model, as implemented in ns-2.28, provides a diskrange model, i.e., a fix communication and carrier sense ranges for a defined TxPower. The log-normal shadowing model, also from ns-2.28, has been configured with a path loss exponent $\beta=2$ and a shadowing deviation $\sigma_{d B}=6 \mathrm{~dB}$ according to an outdoor environment. The Nakagami model was built in [23] and configured as in [10], with a fading intensity $m=3$ for distances up to $50 \mathrm{~m}, \mathrm{~m}=1.5$ for distances between $50 \mathrm{~m}$ and $150 \mathrm{~m}$, and $\mathrm{m}=1$ for distances larger than $150 \mathrm{~m}$.

We remark the importance of performing simulations with propagation models that include realistic (shadowing and fading) effects, in order to estimate the performance results with a higher degree of accuracy. In Fig. 6, we report the probability of correctly receiving a message in absence of interference as a function of distance, for the three propagation models considered in our simulations.

Note that the term communication range (CR) does only hold with TRG due to its deterministic approach. In the following though, we will make use of $\mathrm{CR}=250 \mathrm{~m}$ as equivalent to TxPower $=-37.2 \mathrm{~dB}$ and $\mathrm{CR}=500 \mathrm{~m}$ as equivalent to TxPower $=-31.1 \mathrm{~dB}$ for conveniency. The main configuration parameters used in our simulations are reported in Table II.

For statistical significance, we run 50 times each possible

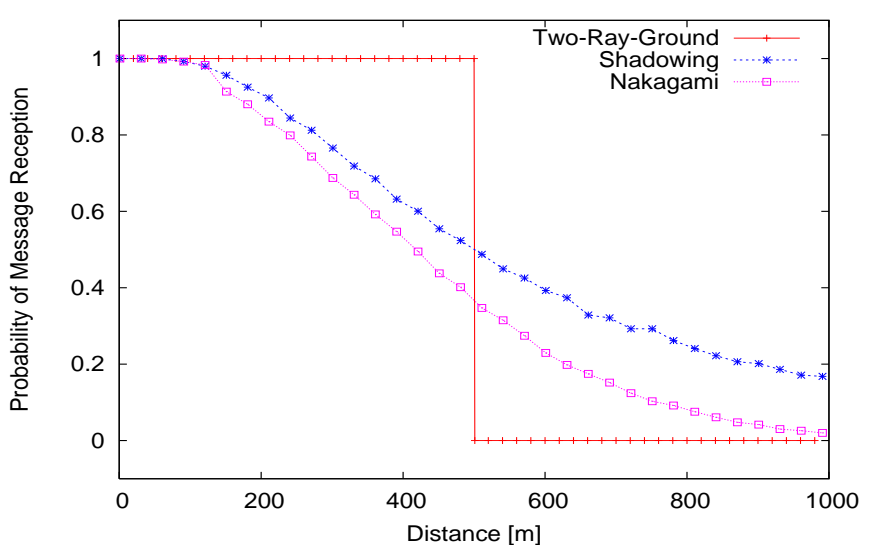

Fig. 6. Probability of correctly receiving a message as a function of distance for the simulated radio propagation models, with $\mathrm{CR}=500 \mathrm{~m}$ $($ TxPower $=-31.1 \mathrm{~dB})$.

TABLE II

CONFIGURATION PARAMETERS

\begin{tabular}{|l|l|}
\hline $802.11 \mathrm{p}$ data rate & $3 \mathrm{Mbps}$ \\
\hline Packets generation rate & $10 \mathrm{packets} / \mathrm{s}$ \\
\hline Packet size & 500 bytes \\
\hline Intended comm. range & $250 \mathrm{~m}, 500 \mathrm{~m}$ \\
\hline Radio propagation models & $\mathrm{TRG}, \mathrm{Nak}, \mathrm{LNS}$ \\
\hline Number of lanes & $3 \times$ direction \\
\hline Vehicle density & 11 cars $/(\mathrm{km} \cdot$ lane $)$ \\
\hline D-FPAV & On, Off \\
\hline D-FPAV MBL & $1.5 \mathrm{Mbps}$ \\
\hline
\end{tabular}

configuration with different random seeds during 10 seconds of real time. The average and the confidence interval (with $95 \%$ confidence level) of the studied metrics are presented in the following section.

In the highway scenario described above, we have configured all vehicles to send beacon messages with the prescribed rate, and one specific node, which is located approximately in the center of the $12 \mathrm{~km}$ long road, to send event-driven messages. Event-driven messages are sent with the same rate as beacons (10 packets/s) and at maximum transmit power. In order to evaluate D-FPAV performance we perform two types of simulation, D-FPAV-Off and D-FPAV-On. In D-FPAV-Off simulations, beacons are sent at maximum power since no power control is applied. On the other hand, for D-FPAV-On, beacons are sent at the transmit power computed by D-FPAV. Note that when applying D-FPAV, only the beacon's TxPower can be decreased.

The two metrics analyzed to evaluate D-FPAV's performance are: $i)$ the average Channel Busy Time ratio (CBT), and $i$ ) the probability of successful reception of a (beacon and event-driven) message with respect to the distance. CBT represents the fraction of time that a wireless interface senses the channel to be busy, i.e., a possible transmission (with energy higher than its CS threshold) is on the medium. The CBT metric is used to corroborate the claim that D-FPAV reduces the load on the channel uniformly in the network, i.e., it achieves fairness. The probability of reception is used 
to assess D-FPAV's effectiveness in achieving an appropriate prioritization of safety-related messages (design goal stated in Sec. II-B), which is obtained by increasing the probability of correctly receiving event-driven messages while at the same time not decreasing too much the probability of correctly receiving beacons close to the sender.

Due to space restrictions, only figures corresponding to $\mathrm{CR}=500 \mathrm{~m}$ are provided and will be used for the results' discussions. However, the differences with $\mathrm{CR}=250 \mathrm{~m}$ will be remarked when appropriate.

\section{B. Simulation Results}

Figures 7, 8 and 9 depict the average CBT for all nodes in the network when $\mathrm{CR}=500 \mathrm{~m}$ for the different radio propagation models. In the highway scenario, nodes with ID from 0 to 467 correspond to vehicles driving in one direction, and nodes with ID from 468 to 932 correspond to vehicles driving in the opposite direction. Note how vehicles that keep close to the border of the highway sector for the ten simulated seconds (IDs smaller than 60, between 450 and 550, and higher than 900) experience lower CBT due to the border effect.

The following observations are in order:

a) Fairness: If no power control is applied, nodes experience considerably different values of the CBT, ranging from about 0.3 to 0.6 with TRG propagation, and from about 0.3 to 0.7 with LNS propagation. This means that different nodes have different opportunities of sending and correctly receiving event-driven messages, impairing fairness. Even worse, nodes traveling in denser areas, where the likelihood of having an accident is higher, experience a higher CBT, which results in a longer expected delay in propagating event-driven messages. On the other hand, when D-FPAV is active all the nodes (excluding the ones on the border, which have a lower CBT value) have the same CBT value, i.e. the same opportunities of sending and correctly receiving safety messages. In other words, D-FPAV achieves its design goal of fairness.

b) $C B T$ reduction with D-FPAV: independently of the radio propagation model, D-FPAV achieves considerable reductions in the CBT value with respect to the case of no power control. For instance, in case of LNS propagation, the CBT value of certain nodes can be reduced from about 0.7 to about 0.3 , i.e., a $57 \%$ reduction. As will be seen later in this section, the reduction of the average CBT provides the desired bandwidth resources in order to improve the event-driven messages' reception rates at all distances from the sender.

c) Effects of the radio propagation model: in absolute terms, the highest CBT values are experienced with LNS propagation. See the smooth decrease with distance of the probability of correctly receiving a message with LNS propagation in Fig. 6, which implies a larger area being interfered (on the average) when there is an ongoing communication. As we will see later, this phenomenon impacts also the probability of correctly receiving a (beacon or event-driven) message.

The same behavior with respect to CBT can be observed case of $\mathrm{CR}=250 \mathrm{~m}$, the only relevant difference being that smaller reductions in the CBT values when D-FPAV is used

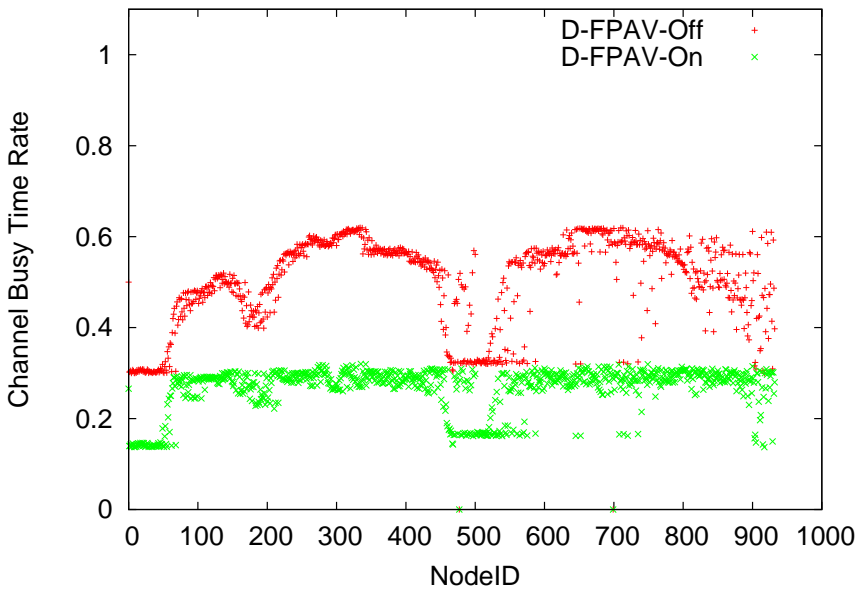

Fig. 7. Channel Busy Time of each node in the highway scenario with Two-Ray Ground model, CR $=500$ and D-FPAV-On/Off.

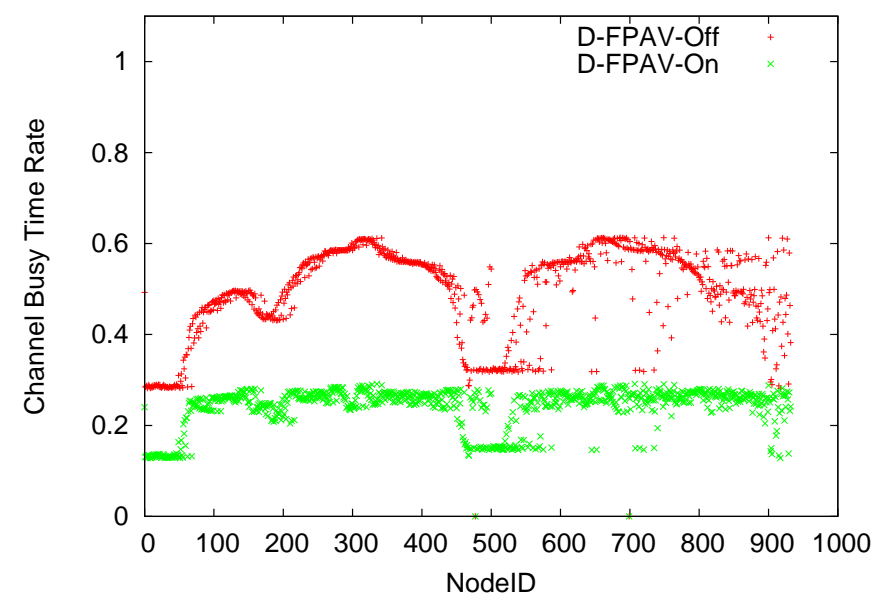

Fig. 8. Channel Busy Time of each node in the highway scenario with Nakagami model, $\mathrm{CR}=500$ and D-FPAV-On/Off.

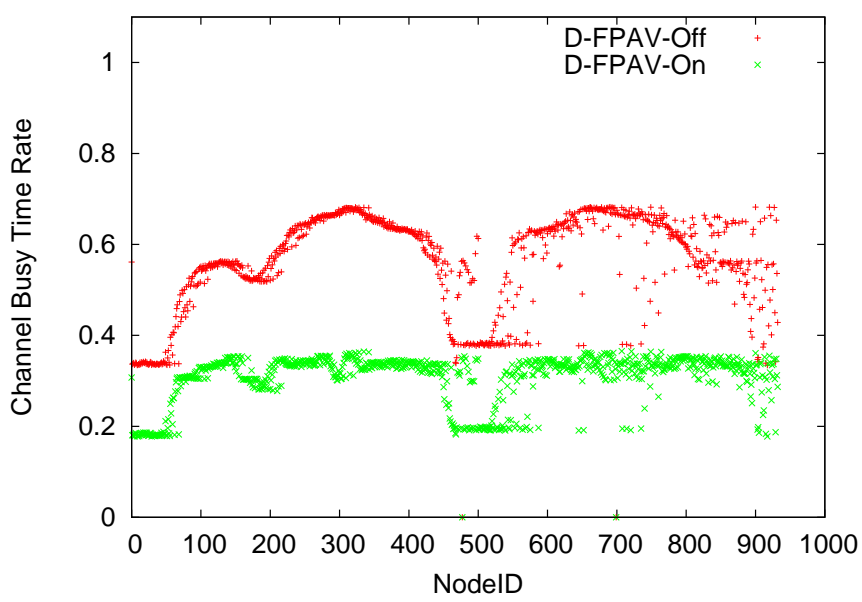

Fig. 9. Channel Busy Time of each node in the highway scenario with Log-Normal Shadowing model, CR = 500 and D-FPAV-On/Off. 
can be observed. For instance, in case of LNS propagation, some nodes experience a reduction in the CBT value from about 0.5 to 0.3 , a $40 \%$ reduction.

Let us now consider the probability of correctly receiving a (event-driven or beacon) message as a function of distance. Figures 10, 11 and 12 report the probability of message reception for all settings described in Section V-A.

The following can be observed from the figures:

a) Higher probability of receiving event-driven messages: independently of the radio propagation model, of the intended communication range, and of the distance from the sender, the probability of correctly receiving an event-driven message with D-FPAV-On is higher than in the case of no power control. The amount of the increase in reception probability depends on the distance from the sender, on the radio propagation model, and on the CR. In general, we can observe an intermediate range of distances (about $100 \mathrm{~m}-250 \mathrm{~m}$ with $\mathrm{CR}=250 \mathrm{~m}$, and about $100 \mathrm{~m}-600 \mathrm{~m}$ with $\mathrm{CR}=500 \mathrm{~m}$ ) in which the increase of the reception probability with D-FPAV is more notable. For instance, with Nak propagation and CR $=500 \mathrm{~m}$, the probability of correctly receiving an event-driven message at a distance of $400 \mathrm{~m}$ from the sender is about 0.42 with D-FPAV, while it is only about 0.2 with no power control, corresponding to a $110 \%$ increase.

b) Lower probability of receiving beacon messages: the price to pay for having a higher probability of receiving eventdriven messages is a decreased probability of receiving beacon messages. The amount of this decrease is strongly dependent on distance: when the receiver is very close to the sender (100m or less), the probability of correctly receiving the beacon is only marginally reduced with respect to the case of no power control. Actually, it is even increased in very close distances to the sender (for instance, with Nak propagation, $\mathrm{CR}=500 \mathrm{~m}$, and distance below $80 \mathrm{~m}$ ). After this 'close in' distance, the probability of correctly receiving beacons is significantly lower than in the case of no power control. This lower beacon reception probability is due to the lower transmit power used to transmit beacons, which is necessary to reduce the beaconing load below the MBL threshold.

c) Effects of the radio propagation model: as expected, the reception probability is lower with more realistic propagation models such as LNS and Nak than in the case of deterministic propagation such as TRG. For instance, the probability of correctly receiving an event-driven message at $200 \mathrm{~m}$ from the sender with $\mathrm{CR}=250 \mathrm{~m}$ is about 0.8 with TRG, while it is about 0.45 with both LNS and Nak. When the CR is doubled, the reception probabilities at $200 \mathrm{~m}$ become about 0.92 with TRG, and about 0.75 with LNS and Nak.

We want to remark that the observed behavior with D-FPAV-On (higher reception probability for event-driven messages at all distances, and lower reception probability for beacons at medium-long distances) satisfies the safetyrelated design goals outlined in Sec. II-B. Beacons are repeated frequently, and the information they convey is mostly important for nodes in close distances to the sender. Hence, the lower reception probability of beacons beyond the 'close in'

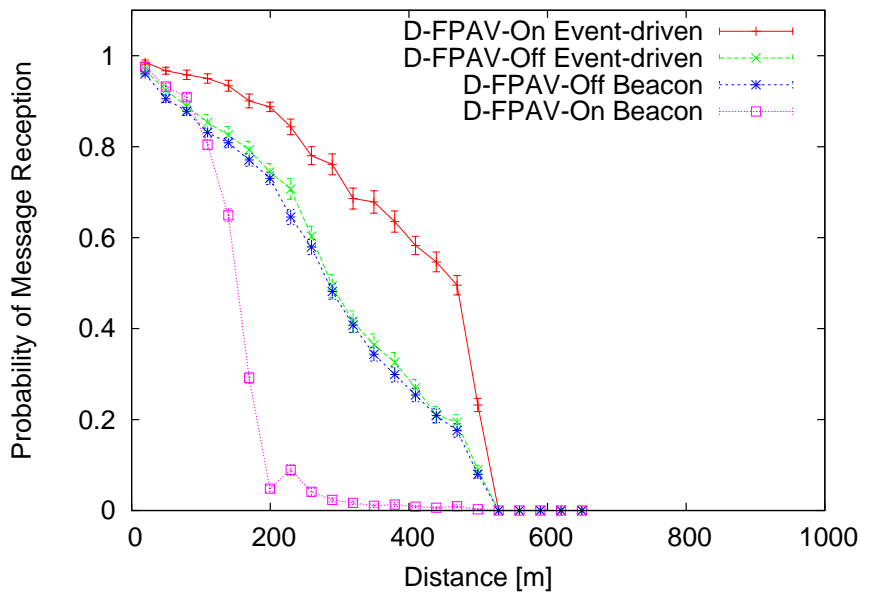

Fig. 10. Probability of Reception over the distance with Two-Ray Ground model, $\mathrm{CR}=500$ and D-FPAV-On/Off.

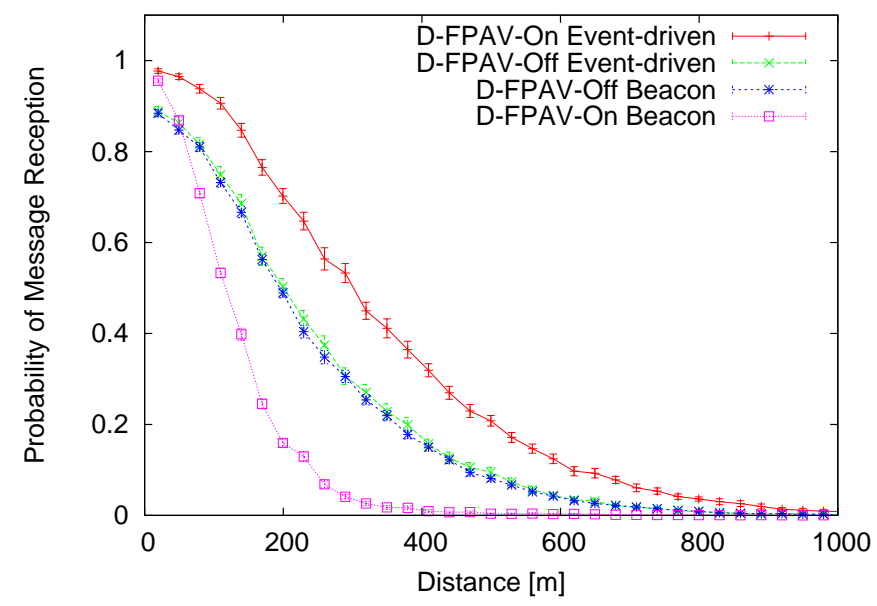

Fig. 11. Probability of Reception over the distance with Nakagami model, $\mathrm{CR}=500$ and D-FPAV-On/Off.

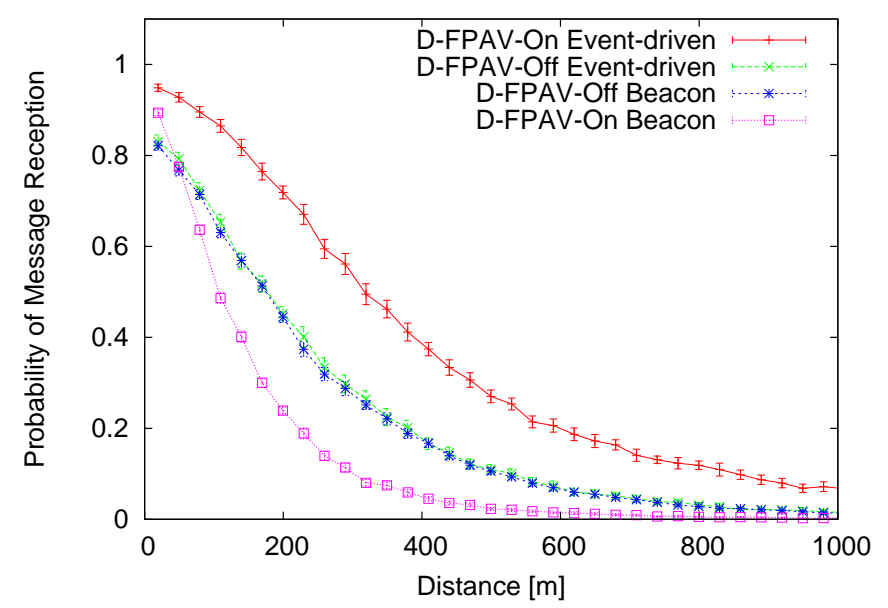

Fig. 12. Probability of Reception over the distance with Log-Normal Shadowing model, $\mathrm{CR}=500$ and D-FPAV-On/Off. 
distance is not critical in terms of safety. On the other hand, event-driven messages require an immediate action at the moment they are issued for vehicles located at both close and further distances, so that accidents can be avoided. This is accomplished by the considerably higher reception probability of event-driven messages with D-FPAV-On, at both close and further distances from the sender.

\section{CONCLUSIONS}

In this paper, we have addressed a major challenge in VANETs, the problem of allocating network resources among neighboring vehicles on the road. Following the guidelines described in [12], we have classified VANETs safety-related messages in event-driven and beacons, and discussed their relevance with respect to the distance to the sender. Motivated by expected channel saturation conditions in high traffic scenarios, we have proposed a fully distributed strategy called $D$-FPAV based on transmit power adjustment of beaconing messages. Contrary to previous power control studies, our strategy is motivated by the safety requirements of VANETs' applications: fairness is the metric to be achieved, coupled with a balance of safety-related messages. We have formally defined the problem and proven that the D-FPAV algorithm achieves fairness. We have conducted a performance evaluation based on ns-2 simulation with a realistic - fast moving 'heavy' traffic density - highway scenario, and considering different radio propagation models (both deterministic and probabilistic). The obtained results show that D-FPAV achieves $i$ ) strict fairness in terms of channel busy time sensed by every node in the highway and ii) a priorization of event-driven messages over beacons. The reception probability of event-driven messages can be increased with D-FPAV at all distances to the sender, while the reception probability of beacons is only marginally reduced for 'close in' distances, i.e., where their information is most relevant.

In terms of future work, we are interested in determining the effect of changing the MBL value on the D-FPAV performance, i.e., fine-tuning the relative priority of event-driven with respect to beacon messages. In turn, we intend to evaluate how the increase in event-driven message reception probability translates into a faster propagation of hazard warnings on the road.

\section{ACKNOWLEDGMENT}

Marc Torrent-Moreno acknowledges the support of the German Ministry of Education and Research $(\mathrm{BMB}+\mathrm{F})$ for the 'Network on Wheels' project under contract number 01AK064F. The authors thank Jens Mittag for help on the ns-2 simulations, and the anonymous reviewers for the many insightful comments.

\section{REFERENCES}

[1] "White Paper - European transport policy for 2010: Time to Decide," Office for Official Publications of the European Communities, ISBN 92-894-03-41-1, 2001

[2] "Vehicle Infrastructure Integration (VII) project," http://www.its.dot.gov/ vii/.
[3] "Car2Car Communication Consortium," http://www.car-to-car.org/.

[4] "Internet ITS Consortium," http://www.internetits.org.

[5] "Dedicated Short Range Communications working group," http:// grouper.ieee.org/groups/scc32/dsrc/index.html.

[6] "Federal Communications Commision. FCC 03-324. FCC Report and Order," February 2004.

[7] "IEEE P802.11p/D0.21, Draft Amendment to Standard for Information Technology Telecommunications and Information Exchange Between Systems LAN/MAN Specific Requirements Part 11: Wireless LAN Medium Access Control (MAC) and Physical Layer (PHY) Specifications: Wireless Access in Vehicular Environments (WAVE)," January 2006.

[8] J. Maurer, T. Fügen, and W. Wiesbeck, "System Simulations Based on IEEE 802.11a for Inter-Vehicle Communications Using a Realistic Channel Model," in Proceedings of the 2nd International Workshop on Intelligent T ransportation (WIT), Hamburg, Germany, March 2005.

[9] M. Raya and J. Hubaux, "The Security of Vehicular Ad Hoc Networks," in Proceedings of the third ACM Workshop on Security of Ad Hoc and Sensor Networks (SASN), Alexandria, Virginia, USA, November 2005.

[10] M. Torrent-Moreno, D. Jiang, and H. Hartenstein, "Broadcast Reception Rates and Effects of Priority Access in 802.11-Based Vehicular Ad-Hoc Networks," in Proceedings of the 1st ACM International Workshop on Vehicular Ad Hoc Networks (VANET), Philadelphia, Pennsylvania, USA, October 2004.

[11] Q. Xu, T. Mak, J. Ko, and R. Sengupta, "Vehicle-to-Vehicle Safety Messaging in DSRC," in Proceedings of the 1st ACM International Workshop on Vehicular Ad Hoc Networks (VANET), Philadelphia, Pennsylvania, USA, October 2004

[12] M. Torrent-Moreno, P. Santi, and H. Hartenstein, "Fair Sharing of Bandwidth in VANET," in Proceedings of the 2nd ACM International Workshop on Vehicular Ad Hoc Networks (VANET), Cologne, Germany, September 2005.

[13] D. Bertsekas and R. Gallager, Data Networks. Prentice Hall, 1987.

[14] M. Kubisch, H. Karl, A. Wolisz, L. Zhong, and J. Rabay, "Distributed Algorithms for Transmission Power Control in Wireless Sensor Networks," in Proceedings of IEEE Wireless Communications and Networking Conference (WCNC), New Orleans, LA, USA, March 2003.

[15] V. Kawadia and P. Kumar, "Principles and Protocols for Power Control in Wireless Ad Hoc Networks," IEEE Journal on Selected Areas in Communications (JSAC), January 2005.

[16] X. Li, T. Nguyen, and R. Martin, "Using Adaptive Range Control to Maximize 1-Hop Broadcast Coverage in Dense Wireless Networks," in Proceedings of the 1st IEEE Conference on Sensor and Ad Hoc Communications and Networks (SECON), Santa Clara, CA, USA, October 2004.

[17] Z. Fang and B. Bensaou, "Fair Bandwidth Sharing Algorithms based on Game Theory Frameworks for Wireless Ad-hoc Networks," in Proceedings of the 23rd Conference of the IEEE Communications Society (INFOCOM), March 2004.

[18] X. Gao, T. Nandagopal, and V. Bharghavan, "Achieving Application Level Fairness Through Utility-based Wireless Fair Scheduling," in Proceedings of the IEEE Global Communications Conference (GLOBECOM), November 2001.

[19] M. Artimy, W. Robertson, and W. Phillips, "Assignment of Dynamic Transmission Range Based on Estimation of Vehicle Density," in Proceedings of the 1st ACM International Workshop on Vehicular Ad Hoc Networks (VANET), Philadelphia, Pennsylvania, October 2004.

[20] L. Wischhof and H. Rohling, "On Utility-Fair Broadcast in Vehicular Ad Hoc Networks," in Proceedings of the 2nd International Workshop on Intelligent Transportation (WIT), Hamburg, Germany, March 2005.

[21] "Network Simulator ns-2," http://www.isi.edu/nsnam/ns/.

[22] F. Schmidt-Eisenlohr, J. Letamendia-Murua, M. Torrent-Moreno, and H. Hartenstein, "Bug Fixes on the IEEE 802.11 DCF module of the Network Simulator ns-2.28," Department of Computer Science, University of Karlsruhe, Tech. Rep. TR-2006-1, January 2006.

[23] V. Taliwal, D. Jiang, H. Mangold, C. Chen, and R. Sengupta, "Empirical Determination of Channel Characteristics for DSRC Vehicle-to-vehicle Communication," in Proceedings of the 1st ACM International Workshop on Vehicular Ad Hoc Networks (VANET), Philadelphia, Pennsylvania, October 2004.

[24] H. Füßler, M. Torrent-Moreno, R. Krüger, M. Transier, H. Hartenstein, and W. Effelsberg, "Studying Vehicle Movements on Highways and their Impact on Ad-hoc Connectivity," Department of Computer Science, University of Mannheim, Tech. Rep. TR-2005-03, June 2005. 